\title{
Special issue on computing, communication and sensor network: CCSN, Puri, Odisha, India, 2014
}

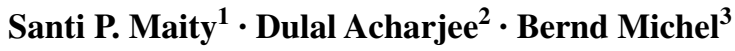

Published online: 21 February 2017

(C) Springer-Verlag Berlin Heidelberg 2017

Recent years have witnessed a prolific growth in the fields of Computing, Communication and Sensor Networks (CCSN) that inspire us to organize International Conference CCSN in every year since 2012 to aware the researchers working worldwide about the latest development in these research fields; CCSN 2014 was the third edition held on 12-14th December, 2014 at Puri, Odisha, India. There were total 51 papers presented on the 2 days of the conference, out of which 18 papers were selected as an extended version (at least $40 \%$ as new content and contribution with respect to its conference version) submission in the MST Journal, Springer. Each extended version was then blind reviewed by two-three domain experts of the respective research fields across the globe and finally based on their recommendations total 11 papers were selected for publication in the MST journal.

The first paper was "Miniature on-chip bandpass filter for RF applications" by B. V. N. S. M. Nagesh Deevi and N. B. Rao. The paper proposes the VLSI multi-layer on chip design for inductor and capacitor as series LC bandpass filter. The second paper was "FPGA based area efficient RS $(23,17)$ codec" by J. Samanta, J. Bhaumik and S. Barman. A compact RS $(23,17)$ encoder and decoder circuit is designed and implemented for UWB applications.

Santi P. Maity

santipmaity@it.iists.ac.in

Bernd Michel

bernd.michel@coinn.de

1 Indian Institute of Engineering Science and Technology, Shibpur, India

2 International Association of Science, Technology and Management, Kolkata, India

3 Micro Materials Center Berlin, Berlin, Germany
The third paper was "Design of an efficient phase frequency detector to reduce blind zone in a PLL" by U. Nanda, D. P. Acharya and S. K. Patra. The paper proposes a novel technique to reduce the blind zone by incorporating a variable delay element. The forth paper "Ultra high gain CMOS Op-Amp design using self-cascoding and positive feedback" authored by S. Chakraborty, A. Pandey and V.Nath suggests a novel design to offer enhanced gain in two stage CMOS amplifier.

The fifth paper entitled "DyProSD: A dynamic protocol specific defense for high-rate DDoS flooding attacks" authored by D. Boro and D. K. Bhattacharyya proposes a defense solution to handle high rate distributed denial of service (HDDoS) flooding attack in Internet. The sixth paper "Enhancing the delivery with density controlled clustering in wireless sensor networks" by G. Raval, M. Bhavsar and N. Patel discusses the performance studies of different clustering protocols in WSN with respect to the network lifetime, data delivery and energy consumption.

The ease of implementation through inclusion of spatial domain features with low computation cost of iterative back projection (IBP) is exploited for super resolution image re-construction in the paper "An edge preserving IBP based super resolution image reconstruction using $\mathrm{P}$-spline and MuCSO-QPSO algorithm" authored by R. Nayak and D. Patra. The eighth paper "Hidden Markov model a tool for recognition of human contexts using sensor of smart mobile phone" by D. Acharjee, S. P. Maity and A. Mukherjee proposes an activity recognition system based on Hidden Markov Model (HMM) using inbuilt sensors of smart mobile phones.

The paper "Wide range-low jitter PLL design for serializer" authored by J. K. Ravia, M. V. Shah, H. Gupta,S. Mehta and A. R. Chowdhury presents the wide range phase locked loop (PLL) design that offers $99.2 \%$ linearity over 
wide frequency range from $14 \mathrm{MGz}$ to $1.05 \mathrm{GHz}$. The paper "Sustainable life-span of WSN nodes using participatory devices in pervasive environment "by N. Prabakaran and R. J. Kannan discusses on resource sharing for reliable communication using relay nodes at the event of node failure. The last paper "Physical layer design for image transmission between WiMAX/DSRC system" by V. D. Kumar, D. Kandar and B. S. Paul critically explores the frame structure and packet format in a hybrid infrastructure for efficient vehicular communication.

On behalf of the organizing and program committee of the conference, we would like to thank the authority of
MST, Springer, to provide us opportunity to work as editor of the special issue. Also thanks to all reviewers of the articles for their esteem effort and time to uphold the spirit of the special issue of the Springer Microsystem Technology Journal. Finally it is also observed that the published papers cover the research fields of computing, communication, sensor network and hardware implementation works meeting the goals of conference like CCSN. We hope the articles will be useful to the research community to put forward the progress in the respective and the allied research fields. 